\title{
Transfer learning with Resnet-50 for detecting COVID-19 in chest X-ray images
}

\author{
Fatima-Zohra Hamlili, Mohammed Beladgham, Mustapha Khelifi, Ahmed Bouida \\ Information Information Processing and Telecommunication Laboratory (LTIT), Department of Electrical Engineering, \\ Faculty of Technology, University TAHRI Mohammed, Bechar, Algeria
}

\begin{tabular}{l} 
Article Info \\
\hline Article history: \\
Received Aug 13, 2021 \\
Revised Dec 21, 2021 \\
Accepted Jan 6, 2022 \\
\hline Keywords: \\
Chest X-ray images \\
Classification \\
COVID-19 \\
Deep-learning \\
Pre-trained deep CNN model
\end{tabular}

\begin{abstract}
The novel coronavirus, also known as COVID-19, initially appeared in Wuhan, China, in December 2019 and has since spread around the world. The purpose of this paper is to use deep convolutional neural networks (DCCN) to improve the detection of COVID-19 from X-ray images. In this study, we create a DCNN based on a residual network (Resnet-50) that can identify COVID-19 from two other classes (pneumonia and normal) in chest X-ray images. DCNN was evaluated using two classification methods: binary (BC-1: COVID-19 vs. normal, BC-2: COVID-19 vs. pneumonia) and multi-class (pneumonia vs. normal vs. COVID-19). In all experiments, four fold cross-validation was used to train and test the model. This architecture's average accuracy is $99.9 \%$ for BC-1, $99.8 \%$ for BC-2, and $97.3 \%$ for multiclass cases. The experimental findings demonstrated that the suggested system detects COVID-19 with an average precision and sensitivity of 95\% and $95.1 \%$ for multi-class classification, respectively. According to our findings, the proposed DCNN may help health professionals in confirming their first evaluation of COVID-19 patients.
\end{abstract}

This is an open access article under the CC BY-SA license.

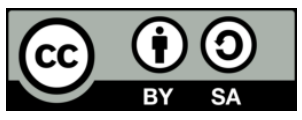

\section{Corresponding Author:}

Fatima-Zohra Hamlili

Information Processing and Telecommunication Laboratory (LTIT), Department of Electrical Engineering

Faculty of Technology, University TAHRI Mohammed

Bechar 08000-Algeria

Email: hamlili.fatima@univ-bechar.dz

\section{INTRODUCTION}

By the end of 2019, a novel virus identified as COVID-19 had appeared in Wuhan, China. Within a few months, the virus had spread all across the world [1], [2]. COVID-19 is a dangerous virus that causes severe symptoms in humans, including cough (76\%), fever (98\%), and fatigue (44\%). The World Health Organization (WHO) announced a coronavirus pandemic on March 11, 2020 [3]. COVID-19 disease is a virus family that includes SARS and respiratory syndrome (MERS) [4].

SARS-CoV first arrived in 2003, infecting 8098 persons with a 9\% death rate across 26 countries, whereas MERS first appeared in 2012, with 688 confirmed cases and 282 deaths in 20 countries [5], [6]. Chest radiography images (CXR and CT scan images) are extremely significant and widely used techniques for classifying diseased lungs [7]. The most popular and commonly available imaging method is the chest Xray, which is used in both emergency and hospital settings [8].

In the context of medical big data analysis, many classic algorithms have been studied, such as logistic regression [9], random forest algorithm [10], and support vector machine [11]. Through the development of deep learning, continuous success has been achieved [12]. Deep learning approaches have proven to be highly successful for a wide range of big data studies, and have therefore been a subject of research interest due to their better performance in learning feature representations from raw data in an end- 
to-end way. Because hidden layers may capture nonlinear connections, adding more hidden layers to a neural network allows it to represent more complicated hypotheses [13]. With the availability of large data, the features extracted from data have increased expressive power; hence, deep learning enables clinical data to be studied in the direction of precision medicine [14].

With the global spread of COVID-19, many studies have used classical machine learning algorithms for efficient COVID-19 identification from X-ray images, including the method described in [15], which is based on multilayer thresholding and SVM. The system segmented images of size $512 \times 512$ using a multilevel threshold based on the Otsu objective and then identified them as infected or non-infected using a support vector machine. The model has a high degree of accuracy of $97.48 \%$. The diagnosis of patients with COVID-19 on the basis of machine learning (ML) and the internet of things (IoT) has found in the study [16]. The authors compared the performance of three machine learning (ML) models, namely support vector machine (SVM), random forest (RF), and Naive Bayes (NB), on three COVID-19 diagnosis methodological scenarios, including those based on original and normalized datasets, as well as those based on feature selection. Their SVM model has the highest accuracy of 95\%. In a study published in [17], the authors examined texture and form descriptors for feature extraction using classical classifiers in order to identify COVID-19 from bacterial and viral pneumonia on X-ray images. They compared Multilayer perceptrons, Support vector machines (SVM), decision trees, Bayesian network, and naive Bayes classification algorithms. With an average accuracy of $89.78 \%$, they discovered that SVM performed the best.

Recently, deep learning (DL) has seen increased success in the analysis and interpretation of medical imaging [8]. Many researchers from all around the world are interested in using DL techniques to classify COVID-19, including the work published in [18], in which the authors developed COVID-CheXNet for identifying COVID-19 from chest X-ray images. The main goal of the system is to combine the results of two different deep learning algorithms (HRNet and ResNet34) to distinguish between healthy and COVID-19 infected patients. COVID-CheXNet has a $99.99 \%$ accuracy rate.

Al-Waisy et al. [19] the researchers describe a COVID-DeepNet system, which is a hybrid COVID19 detection system. COVID- DeepNet is comprised of two distinguish deep learning algorithms (DBN and CDBN) that can distinguish between normal and COVID-19 infected cases from X-ray images with a 99.93\% detection rate. COVID-Net, developed by the authors of research [20], was employed to classify normal, pneumonia (bacterial and viral), and COVID-19 patients from chest X-ray images, with an accuracy of $83.5 \%$.

Ozturk et al. [21] proposed a unique DarkNet model for 2-class (no-findings vs. COVID-19) and 3class classification (no-findings vs. COVID-19 vs. pneumonia). The method has a 2-class classification accuracy of $98.8 \%$ and a 3-class classification accuracy of $87.2 \%$. The novel algorithm called OptCoNet presented in [22] is made up of optimized feature extraction and classification components. Using a publicly available dataset of normal, COVID-19, and pneumonia images, the model was evaluated and compared to several classification algorithms. OptCoNet's accuracy was 97.78\%. CoroNet is a method proposed in [23] that is based on pre-trained exception architecture for diagnosing COVID-19 infection in chest X-ray images and has an overall accuracy of $89.6 \%$. Abbas et al. [24], a deep CNN called DeTraC was developed to identify COVID-19 in chest X-ray images. The technique is based on the addition of pre-trained models to class decomposition layer. DeTraC achieved a high accuracy of $98.23 \%$ using pre-trained VGG19. To distinguish between COVID-19 infected and healthy patients in X-ray images, the authors in [25] compared two successful moderns: classical machine learning methods (SVM, ANN, k-NN, RBF, CN 2, and DT) and deep learning models (ResNet50, MobileNetsV2, GoogleNet, Xception, and DarkNet). They observed that ResNet50 had the greatest performance of the four deep learning models, with an accuracy of $98.8 \%$. In comparison, the SVM attained the best accuracy of $95 \%$ in classical machine learning approaches.

Alyasseri et al. [26] completed a comprehensive review of over 200 studies published between December 2019 and April 2021. They observed that COVID-19 diagnosis and epidemic prediction are performed using machine learning methods (LDA, SVM, KNN, Boost, ANN, RF, LR, and K-means) and deep learning techniques (CNN, DNN, RNN, and GANs). They come to the conclusion that SVM is the most generally used machine learning mechanism, whereas CNN is the most often employed deep learning mechanism.

In this paper, COVID-19 was distinguished from two other classes (normal and pneumonia) in chest X-ray images using a deep convolutional neural network (DCNN) based on a pre-trained model Residual Network (ResNet-50) [27]. The performance of our system was examined using two classification methods: binary (BC-1: normal vs. COVID-19 and BC-2: pneumonia vs. COVID-19) and multi-class (normal vs. COVID-19 vs. pneumonia). The results were then compared to previous research published in the literature. Clinicians may find the model useful in diagnosing COVID-19 infection from CXR images.

The remaining sections of this study are organized as follows: Section 2.1 describes the dataset that was used. Sections 2.2, 2.3, and 2.4 discuss preprocessing methodologies, the proposed Deep Convolutional Neural Network, and evaluation metrics, respectively. Section 3 contains a detailed presentation of the 
experimental results. Section 4 contains a discussion and result comparison of our suggested model with current methodologies. Section 5 concludes the article.

\section{RESEARCH METHOD}

In this section, we will introduce the dataset and the approaches used for dataset preprocessing, followed by data augmentation processes. Then, we'll go over the specifics of the deep convolutional neural network (DCNN) proposed in this study. Finally, we will present the benchmark evaluation measures that we used to evaluate the performance of our model, such as precision, specificity, F1-score, sensitivity, and accuracy.

\subsection{Chest $X$-ray dataset}

In our work, we merged datasets from two different sources. The first dataset consists of CXR images of COVID-19 obtained from the open-source GitHub repository [28]. This dataset contains 317 frontal X-ray images of COVID-19 positive cases. The second dataset is from the Kaggle repository "Chest X-ray images (pneumonia)" [29], and it comprises 1583 normal, 1493 viral pneumonia, and 2780 bacterial pneumonia cases with a total of 5856 images. The Figures 1(a)-(d) show some samples of X-ray images.

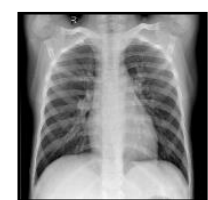

(a)

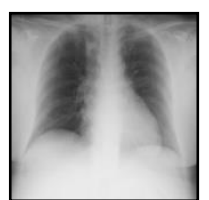

(b)

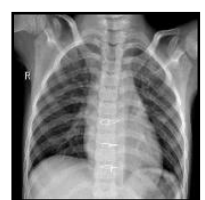

(c)

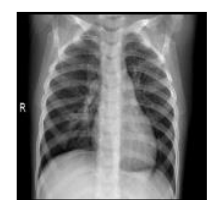

(d)

Figure 1. Samples of X-ray dataset of (a) normal, (b) COVID-19, (c) bacterial pneumonia, and (d) viral pneumonia

\subsection{Dataset pre-processing}

In this work, a preprocessing stage was employed to increase the efficacy of training the classifier, which included two approaches to improving the quality of the original images and removing undesirable characteristics (see Figure 2). To begin, all images were resized to $224 \times 224 \times 3$ to save processing time and make them compatible with ResNet-50 [27]. The low image contrast and small details, textures of the X-ray image were then improved by using an adaptive contrast algorithm (CLAHE). Finally, the wavelet image denoising approach was used to remove the noise from the image created in the previous stage.

\subsubsection{Histogram equalization with adaptive histograms (CLAHE)}

Limited adaptive histogram equalization in contrast (CLAHE) is a modification of the histogram equalization approach (AHE) that was first designed to improve low contrast medical images [30], [31]. Edges and curves in each section of an image may be made more visible with the CLAHE method [32]. The CLAHE technique produces results that are substantially impact by two factors: the number of sub-images and the Clip Limit. The input images are divided into sub-images (tiles) [33]. This division results in three different sets of regions: the corner region (CR), the border region (BR), and the inner region (IR); the contrast transform function is calculated for each tile using the contrast factor 'Clip Limit' [34]. In this research, we apply the CLAHE enhancement approach with Clip Limit 0.1 to impose a limit on a noisy image, and the contrast transform function is created using an exponential distribution parameter to get better results, as shown in (1):

$$
g=g_{\text {min }}-\left(\frac{1}{\alpha}\right) * \ln [1-P(f)]
$$

where $g_{\min }$ defines a minimum pixel value, $\alpha$ is a clip parameter, and $P(f)=\mathrm{CPD}$ (Cumulative probability distribution).

\subsubsection{De-noising algorithm}

The image-de-noising algorithm, as shown in Figure 2, is summarized in the following steps: i) Apply DWT on noisy image up to two levels $(\mathrm{L}=2)$ to split it into seven sub bands (HH1, LH1, HL1, HH2, LH2, HL2 and LL2) by using orthogonal wavelet of Daubechies; ii) Compute noise variance $\sigma$ using (2),

$$
\sigma=\frac{\operatorname{median}\left(\left|x_{i, j}\right|\right)}{0.6745}
$$


where $x_{i, j}$ represents the detail coefficients at the finest level; iii) Calculate the threshold value $\mathrm{T}$ for each pixel by using the universal threshold function as shown in (3),

$$
\mathrm{T}=\sigma \sqrt{2 \log N}
$$

where $\mathrm{N}$ is the image's pixel density; iv) Threshold all sub band coefficients using soft-thresholding function, as in (4),

$$
\mathrm{T}_{\mathrm{t}}^{\text {Soft }}=\left\{\begin{array}{cl}
\operatorname{sgn}(Y[m, n])(|Y[m, n]|-T) & |Y[m, n]|>T \\
0 & |Y[m, n]| \leq \mathrm{T}
\end{array}\right.
$$

where $T_{t}^{\text {Soft }}$ represents soft thresholding, $Y[m, n]$ is a wavelet coefficients, $T$ is the threshold value, and $\operatorname{sgn}(x)$ is the sign function of $\mathrm{x}$; v) Finally, applied the inverse DWT to reconstruct the de-noised image.

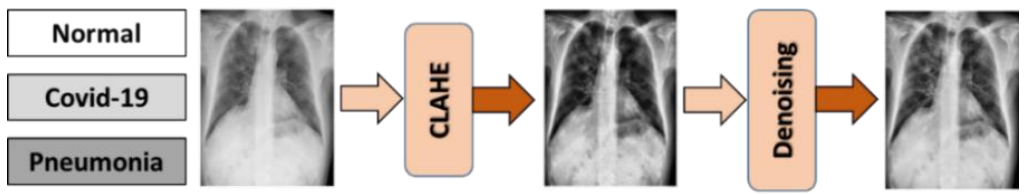

Figure 2. Schematic representation of preprocessing steps to obtain the final images for the dataset

Following the preprocessing step, data augmentation techniques frequently employed in the medical area were utilized to enhance the number of samples in the constrained dataset by creating additional tagged images while keeping the semantic meaning of the images. This work employs the following transformations: horizontal flip, rotation, and translation. To avoid biased prediction results, data augmentation was applied after partitioning the dataset into four cross validations.

\subsection{Proposed deep convolutional neural network with ResNet-50}

In recent years, transfer learning (TL), which is a good feature extractor, has become more popular in medical imaging applications. The use of a pre-trained model to classify images is common in the design of TL. Among the numerous deep learning models available, the residual neural networks (Resnet-50) created by He et al. [27] was used in this study.

In this paper, we created a deep CNN based on a ResNet-50, followed by 7 additional tasks. The convolution layers of the Resnet-50 model were employed as a base network in the new architectures to generate the feature values for each image, and the additional layers replaced the last 1000 fully connected softmax layer of the ResNet-50 to categorize COVID-19 X-ray images. Three fully connected layers, three dropout layers, and a softmax layer comprise the classification stage. The first two sets of fully connected layers in this architecture have sizes of 200 and 300, respectively, while the last one has a size defined by the number of categories in the dataset. Dropout ratios of $0.12,0.2$, and 0.15 were used. The architecture's dropout layer aids in the alleviation of overfitting and enhances model performance. The softmax activation function was also used to classify X-ray images into two and three cases. A deep convolutional neural network (DCNN) is seen schematically in Figure 3.

\subsection{Performance metrics}

The effectiveness of the proposed approach was measured using many measures, including, Accuracy, F1- score, sensitivity [35], precision, and specificity [36], as shown in (5) to (9),

$$
\begin{aligned}
& \text { Accuracy }=\frac{T N+T P}{T N+T P+F P+F N} \\
& \text { F1- score }=2 \times \frac{\text { Recall } \times \text { Precision }}{\text { Recall }+ \text { Precision }} \\
& \text { Sensitivity }=\frac{T P}{F N+T P} \\
& \text { Precision }=\frac{T p}{F P+T p} \\
& \text { Specificity }=\frac{T N}{T N+F P}
\end{aligned}
$$

where: 
- True Positive (TP): The number of COVID-19 cases that were correctly identified.

- False Negative (FN): The number of COVID-19 cases identified as normal.

- True Negative (TN): The number of cases that were accurately classified as normal.

- False Positive (FP): The number of normal cases classified as COVID-19 cases.

Others metrics of measurement such as $\mathrm{Acc}_{\mathrm{m}}, \mathrm{Sens}_{\mathrm{m}}, \mathrm{Spec}_{\mathrm{m}}, \mathrm{Prec}_{\mathrm{m}}$, and $\mathrm{F} 1-\mathrm{score}_{\mathrm{m}}$ are used to determine the effectiveness of multi-class classification, as shown in (10) to (14). These metrics represent the multiclass accuracy, specificity, sensitivity, precision, and F1-score measurements, respectively:

$$
\begin{aligned}
& \text { Acc }_{\mathrm{m}}=\frac{T P n+T P c+T P p}{\text { Total samples }} \\
& \text { Sens }_{\mathrm{m}}=\frac{T P c+T P p}{T P c+T P p+F N c+F N p} \\
& \text { Spec }_{\mathrm{m}}=\frac{T P n}{F P c+T P n+F P p} \\
& \text { Prec }_{\mathrm{m}}=\frac{T P c+T P p}{T P c+F P c+T P p+F P p} \\
& \mathrm{~F}_{1-\text { score }_{\mathrm{m}}}=2 \times \frac{\text { Prec }_{m} \times \text { Recall }_{m}}{\text { Prec }_{m}+\text { Recall }_{m}}
\end{aligned}
$$

where:

- TPc denotes correctly identified COVID-19 instances.

- TPp refers to pneumonia instances that have been correctly classified.

- TPn represents correctly classified normal cases.

- FPc denotes healthy cases that are classed as COVID-19.

- FPp denotes healthy cases that are diagnosed as pneumonia.

- FNc and FNp indicate COVID-19 and pneumonia cases classed as normal, respectively.

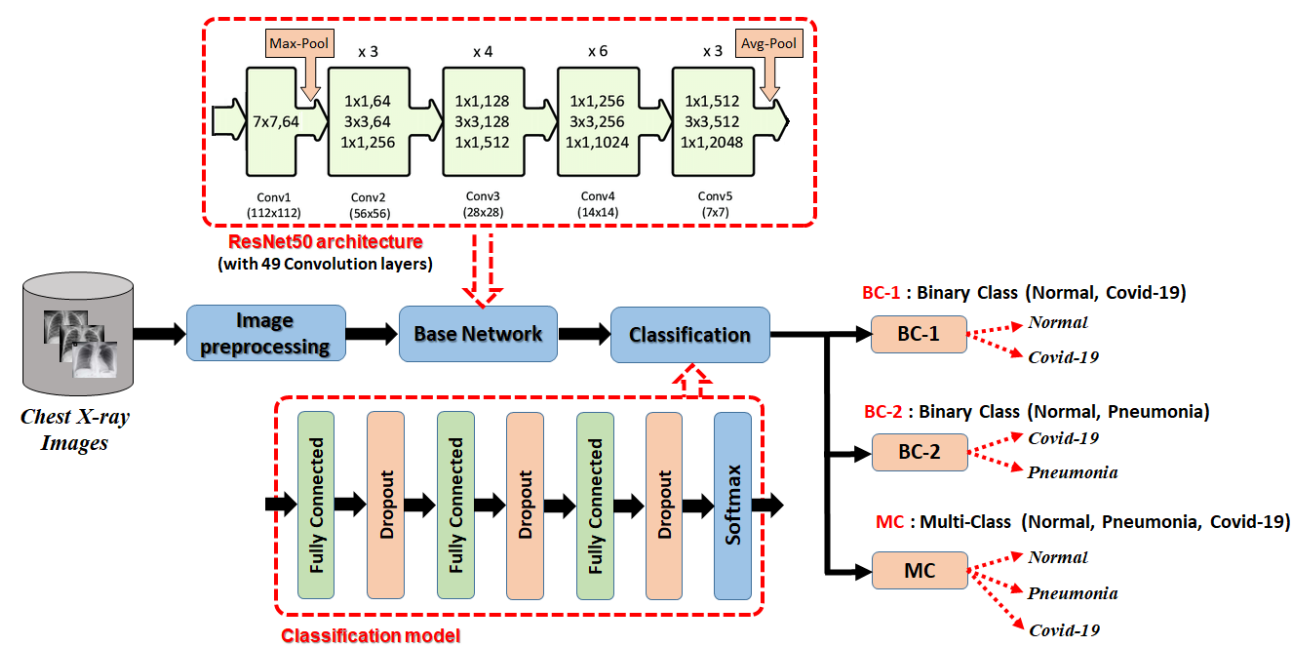

Figure 3. Schematic representation of our suggested deep convolutional neural network (DCNN)

\section{RESULTS}

The experiments were carried out in MATLAB 2020a programming language on a computer with the following configuration: 8 GB of RAM, Intel (R) Core (TM) i5-4460 CPU running, and Windows 10 (64 bit). To improve the efficacy of our model, the proposed DCNN model was trained and evaluated using 4-fold crossvalidation across all experiments. Figure 4 shows four folds cross validation, with three sub-folds chosen for the training and one single sub-fold chosen for the testing. The model was evaluated with two classification methods: binary (BC-1: normal vs. COVID-19 and BC-2: pneumonia vs. COVID-19) and multi-class (normal vs. pneumonia vs. COVID-19). In total, 35 epochs were employed in each fold with a batch size equal to 25 and a learning rate of 0.0001 . The chosen optimization method was the ADAM algorithm for optimizing loss function. 


\begin{tabular}{|c|c|c|c|c|}
\hline 4-Fold CV & \multicolumn{4}{|c|}{ Dataset } \\
\hline Fold-1 & Test & Train & Train & Train \\
\hline Fold-2 & Train & Test & Train & Train \\
\hline Fold-3 & Train & Train & Test & Train \\
\hline Fold-4 & Train & Train & Train & Test \\
\hline
\end{tabular}

Figure 4. Shows a diagram of the 4-fold cross validation technique

\subsection{Experimental results of BC-1 (normal vs. COVID-19)}

The initial classification was binary (normal vs. COVID-19), a total of 1900 images were used (1583 normal, 317 COVID-19). The binary classification performance results for both classes for each fold are shown in Figures 5(a)-(d) as a separate confusion matrix (CM), and the evaluation metrics such as specificity, sensitivity, F1-score, precision, and accuracy, as well as their average values, are reported in Table 1. Specificity, F1-score, precision, and accuracy are all lower in Fold-4, with 98.8\%, 99.8\%, 99.7\%, and 99.8\%, respectively. For all folds, the model, on the other hand, achieved a sensitivity of $100 \%$. Specificity, sensitivity, F1-score, precision, and accuracy have average values of $99.7 \%, 100 \%, 99.9 \%, 99.2 \%$, and 99.9\%, respectively.

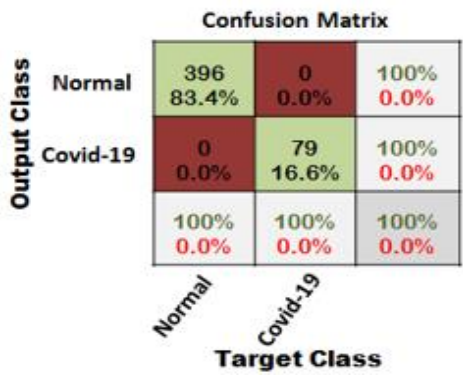

(a)

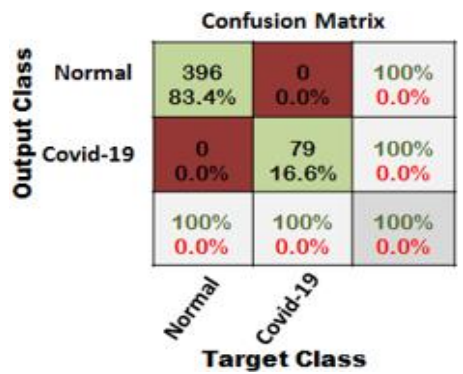

(c)

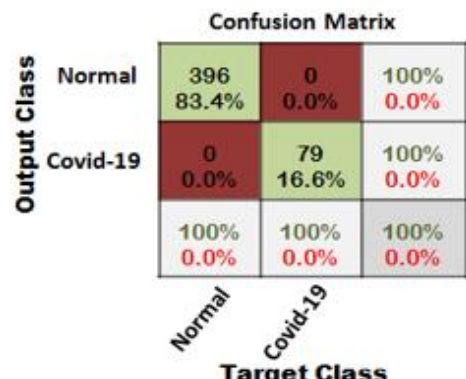

(b)

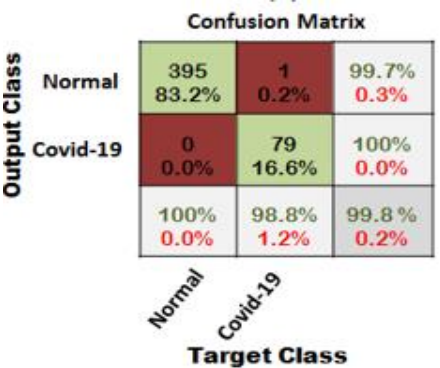

(d)

Figure 5. Confusion matrices of BC-1: (a) Fold-1CM-1, (b) Fold-2CM-2, (c) Fold-3CM-3, and (d) Fold-4CM-4

Table 1. Performance results of binary classification (normal vs. COVID-19) for each fold

\begin{tabular}{cccccc}
\hline \multirow{2}{*}{ Folds } & \multicolumn{5}{c}{ Performance Metrics (\%) } \\
& Sensitivity & Specificity & Precision & f1-score & Accuracy \\
\hline Fold -1 & 100 & 100 & 100 & 100 & 100 \\
Fold- 2 & 100 & 100 & 100 & 100 & 100 \\
Fold- 3 & 100 & 100 & 100 & 100 & 100 \\
Fold -4 & 100 & 98.8 & 99.7 & 99.8 & 99.8 \\
Average & $\mathbf{1 0 0}$ & $\mathbf{9 9 . 7}$ & $\mathbf{9 9 . 2}$ & $\mathbf{9 9 . 9}$ & $\mathbf{9 9 . 9}$ \\
\hline
\end{tabular}

\subsection{Experimental results of BC-2 (COVID-19 vs. pneumonia)}

In this part, the experimental results of the second binary classification (pneumonia vs. COVID-19) are presented. The model was evaluated on a total of 4590 samples (4273 pneumonia and 317 COVID-19). The sensitivity, specificity, F1-score, precision, and accuracy, as well as their average values, are shown in Table 2. On the one hand, Figures 6(a)-(d) show the confusion matrix for each fold. The Fold-4 has a lower sensitivity rate of $99.7 \%$. Furthermore, at Fold-3, the minimum specificity and accuracy values are $94.9 \%$ and $99.7 \%$, respectively. The F1-score for Fold-1 and Fold-2 is 99.9\%. Finally, the maximum accuracy value is $99.9 \%$ at 
Fold-1. The suggested technique achieved average accuracy of 99.8\%, as well as sensitivity, specificity, F1score, and precision values of $99.9 \%, 98.1 \%, 99.9 \%$, and $99.9 \%$, respectively.

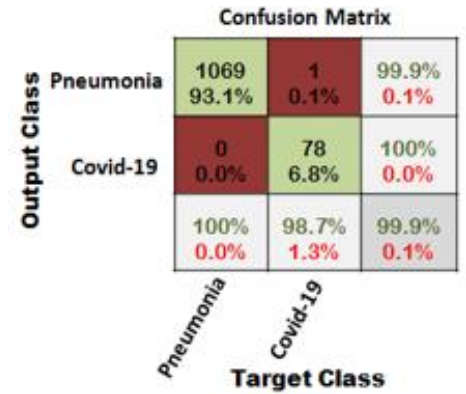

(a)

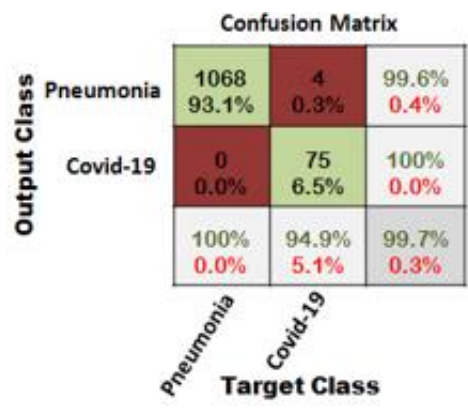

(c)

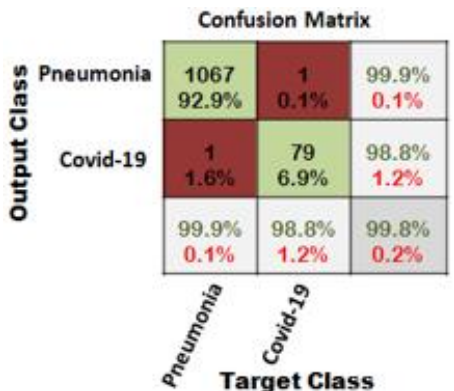

(b)

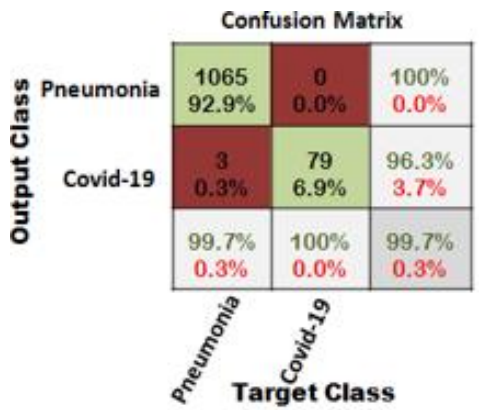

(d)

Figure 6. Confusion Matrices of BC-2: (a) Fold-1 CM-1, (b) Fold-2CM-2, (c) Fold-3CM-3, and (d) Fold-4CM-4

Table 2. Performance results of binary classification (pneumonia vs. COVID-19) for each fold

\begin{tabular}{cccccc}
\hline \multirow{2}{*}{ Folds } & \multicolumn{5}{c}{ Performance Metrics (\%) } \\
& Sensitivity & Specificity & Precision & F1- score & Accuracy \\
\hline Fold- 1 & 100 & 98.7 & 99.9 & 99.9 & 99.9 \\
Fold -2 & 99.9 & 98.8 & 99.9 & 99.9 & 99.8 \\
Fold -3 & 100 & 94.9 & 99.6 & 99.8 & 99.7 \\
Fold- 4 & 99.7 & 100 & 100 & 99.8 & 99.7 \\
Average & $\mathbf{9 9 . 9}$ & $\mathbf{9 8 . 1}$ & $\mathbf{9 9 . 9}$ & $\mathbf{9 9 . 9}$ & $\mathbf{9 9 . 8}$ \\
\hline
\end{tabular}

\subsection{Experimental results of MC (normal vs. COVID-19 vs. pneumonia)}

Finally, the multi-class classification results are presented in this section. A total of 6173 samples (1583 normal, 4273 pneumonias, and 317 COVID-19) were employed to test the effectiveness of the suggested technique on a three-class classification. The evaluation metrics are F1-score sensitivity, specificity, precision, and accuracy and their average values are presented in Table 3. The average F1-score, sensitivity, specificity, precision, and accuracy are $94.9 \%, 95.1 \%, 98.1 \%, 95.0 \%$, and 97.3\%, respectively. Furthermore, at Fold-2, the lowest sensitivity value is $93.9 \%$, while at Fold-4, the maximum sensitivity value is $95.7 \%$. Furthermore, at Fold-4, the specificity value is $97.4 \%$, whereas at Fold-3, it is $98.6 \%$. Furthermore, with Fold-4, the lowest precision and F1-score are $92.9 \%$ and 94\%, respectively, whereas Fold-3 has the greatest values of both. Finally, accuracy varies from $96.8 \%$ at Fold-2 to $97.7 \%$ at Fold-1 and Fold-3. The confusion matrix for each fold is represented in Figure 7(a)-(d).

Table 3. Performance results of the proposed approach in multi-class classification task

\begin{tabular}{lccccc}
\hline \multirow{2}{*}{ Folds } & \multicolumn{5}{c}{ Performance Metrics $(\%)$} \\
& Sensitivity & Specificity & Precision & F1- score & Accuracy \\
\hline Fold- 1 & 95.5 & 98.4 & 95.7 & 95.5 & 97.7 \\
Fold -2 & 93.9 & 97.8 & 94.2 & 94.1 & 96.8 \\
Fold -3 & 95.2 & 98.6 & 97.2 & 96.0 & 97.7 \\
Fold- 4 & 95.7 & 97.4 & 92.9 & 94.0 & 97.0 \\
Average & $\mathbf{9 5 . 1}$ & $\mathbf{9 8 . 1}$ & $\mathbf{9 5 . 0}$ & $\mathbf{9 4 . 9}$ & $\mathbf{9 7 . 3}$ \\
\hline
\end{tabular}




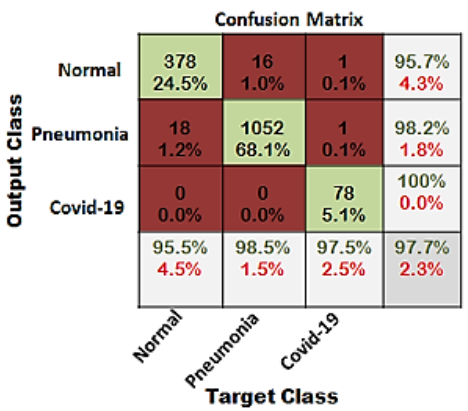

(a)

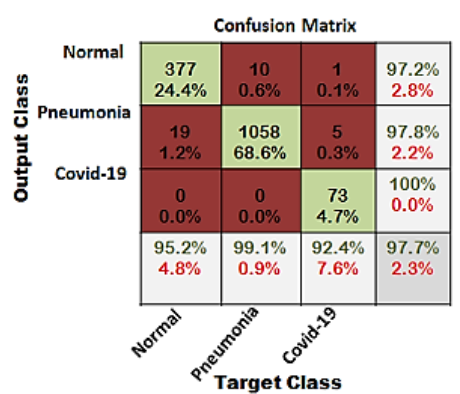

(c)

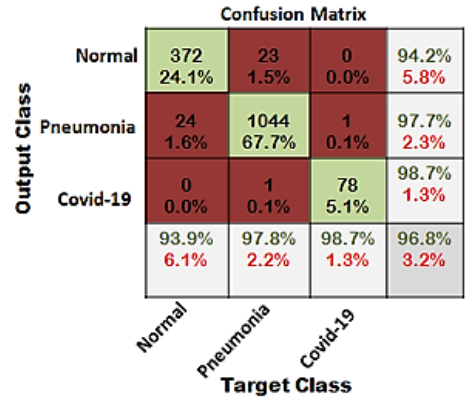

(b)

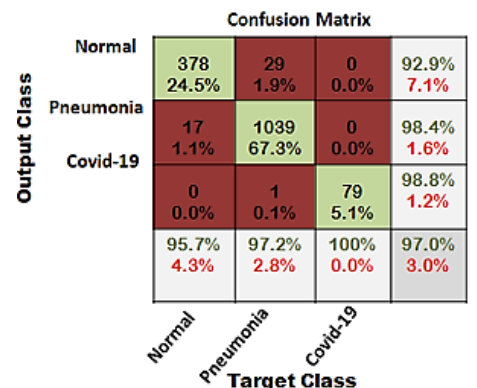

(d)

Figure 7. Confusion Matrices of MC: (a) Fold-1 CM, (b) Fold-2 CM-2, (c) Fold-3 CM-3, and (d) Fold-4 CM-4

\subsection{Experiment 2: final model accuracy evaluation}

To test the robustness of our proposed approach, we used a small dataset produced in [23]. The dataset comprises about 310 normal, 284 COVID-19, 330 pneumonia bacterial, and 327 pneumonia viral chest X-ray images. The experiment used $80 \%$ random selection X-ray images as a training set and $20 \%$ as a testing set. Table 4 shows the acquired results, and Figure 8 illustrates the corresponding confusion matrix. Table 4 demonstrates the sensitivity, F1-score specificity, and precision of our system on dataset-2, as well as their average values. The proposed approach performed well in this experiment, with an overall accuracy of $95.2 \%$.

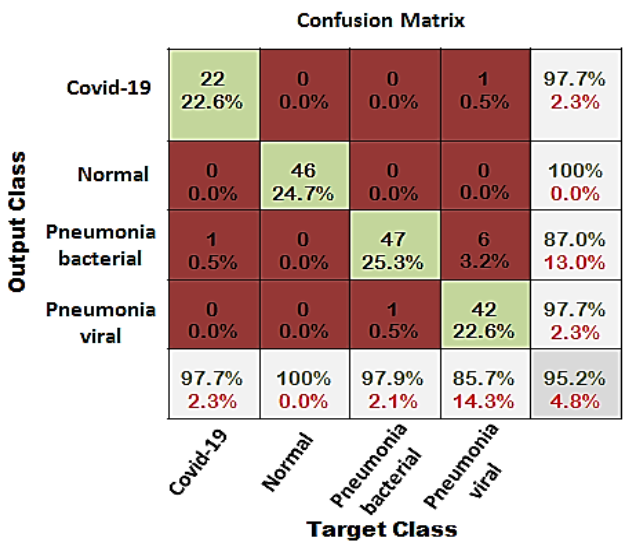

Figure 8. The confusion matrix result of our proposed model on dataset-2

Table 4. Performance evaluation of DCCN model on dataset-2

\begin{tabular}{lcccc}
\hline \multirow{2}{*}{ Class } & \multicolumn{4}{c}{ Performance Metrics (\%) } \\
& Sensitivity & Specificity & Precision & F1- score \\
\hline COVID-19 & 97.7 & 99.3 & 97.7 & 97.7 \\
Normal & 100 & 100 & 100 & 100 \\
Pneumonia Bacterial & 87.0 & 99.2 & 97.9 & 92.1 \\
Pneumonia Viral & 97.7 & 95.1 & 85.7 & 91.3 \\
Average & $\mathbf{9 5 . 6}$ & $\mathbf{9 8 . 4}$ & $\mathbf{9 5 . 3}$ & $\mathbf{9 5 . 3}$ \\
Overall Accuracy & \multicolumn{9}{c|}{$\mathbf{9 5 . 2 \%}$} \\
\hline
\end{tabular}




\section{DISCUSSION}

In this work, deep CNN based on a Resnet-50 was used to detect COVID-19 from chest X-ray images. We have used a total of (6173 X-ray images). Our model has an accuracy of 99.9\%, $99.8 \%$, and 97.3\% for BC-1 (normal vs. COVID-19), BC-2 (pneumonia vs. COVID-19), and MC (pneumonia vs. COVID-19 vs. normal), respectively. The proposed model was also evaluated on another dataset prepared in [23]. The model performed well, with an accuracy of 95.2\%. CoroNet was suggested in [23] as a pretrained Xception architecture-based classification system for four different classes (COVID-19, normal, pneumonia bacterial, and pneumonia Viral). They had an accuracy of $89.6 \%$. Table 5 compares the classification of X-ray images into four categories using CoroNet and our proposed system. According to the results in Table 6, the proposed deep CNN outperforms other methods. The study [24] developed a new method for identifying COVID-19 from X-ray images called DeTraC. They achieved a 93.1\% accuracy rate.

Table 5. Performance comparison of the proposed DCNN model with CoroNet

\begin{tabular}{|c|c|c|c|c|c|c|c|c|}
\hline \multirow[b]{2}{*}{ Class } & \multicolumn{4}{|c|}{ CoroNet } & \multicolumn{4}{|c|}{ Our proposed model } \\
\hline & $\begin{array}{c}\text { Sensitivity } \\
(\%)\end{array}$ & $\begin{array}{c}\text { Specificity } \\
(\%)\end{array}$ & $\begin{array}{c}\text { Precision } \\
(\%)\end{array}$ & $\begin{array}{c}\text { F1-score } \\
(\%)\end{array}$ & $\begin{array}{c}\text { Sensitivity } \\
(\%)\end{array}$ & $\begin{array}{c}\text { Specificity } \\
(\%)\end{array}$ & $\begin{array}{c}\text { Precision } \\
(\%)\end{array}$ & $\begin{array}{c}\text { F1-score } \\
(\%)\end{array}$ \\
\hline COVID-19 & 98.3 & 97.9 & 93.2 & 95.6 & 97.7 & 99.3 & 97.7 & 97.7 \\
\hline Normal & 93.5 & 98.1 & 95.3 & 94.3 & 100 & 100 & 100 & 100 \\
\hline Pneumonia Bacterial & 85.9 & 95.0 & 86.9 & 86.3 & 87.0 & 99.2 & 97.9 & 92.1 \\
\hline Pneumonia Viral & 82.1 & 94.8 & 84.1 & 83.1 & 97.7 & 95.1 & 85.7 & 91.3 \\
\hline Average & 89.9 & 96.5 & 89.8 & 89.8 & 95.6 & 98.4 & 95.3 & 95.3 \\
\hline Overall Accuracy & \multicolumn{4}{|c|}{$89.6 \%$} & \multicolumn{4}{|c|}{$95.2 \%$} \\
\hline
\end{tabular}

Table 6. Shows a comparison of the suggested model to previous research

\begin{tabular}{llcc}
\hline \multicolumn{1}{c}{ Authors } & \multicolumn{1}{c}{ Architecture } & Accuracy two-class (\%) & Accuracy three-class $(\%)$ \\
\hline Mahdy et al. [15] & Multilevel thresholding + SVM & 97.48 & NA \\
Al-Waisy et al. [18] & COVID-CheXNet & 99.99 & NA \\
Wang et al. [20] & Covid-Net & 92.4 & NA \\
Ozturk et al. [21] & DarkNet & 98.08 & 87.02 \\
Goel et al. [22] & OptCoNet & NA & 97.78 \\
Khan et al. [23] & CoroNet & 99 & 89.6 \\
Abbas et al. [24] & DeTraC & NA & 93.1 \\
Our proposed model & DCNN & $\mathbf{9 9 . 9}$ & $\mathbf{9 7 . 3}$ \\
\hline
\end{tabular}

The COVID-DeepNet system was suggested in [20] as a unique hybrid COVID-19 detection approach to detect healthy and COVID-19 diseased patients using X-ray images. The detection accuracy of COVID-DeepNet was $99.93 \%$. The method developed in [15] based on multi-level threshold and SVM, to identify COVID-19 patients from CXR images with a high level of accuracy of 97.48\%. Ozturk et al. [21] proposed the DarkNet model to identify X-ray images using two classification scenarios: two-class (nofindings vs. COVID-19) and three-class (no-finding vs. pneumonia vs. COVID-19). In two-class and threeclass categorization, they attained accuracy of $98.08 \%$ and $87.02 \%$, respectively.

\section{CONCLUSION}

In this work, we develop a deep convolutional neural network (DCNN) system for automatically in this work, we develop a deep convolutional neural network (DCNN) system for automatically discriminating COVID-19 cases from pneumonia and normal cases. Using a pre-trained ResNet-50, the proposed technique was trained and tested on two class and three class classifications. The experimental findings demonstrate that our designed system achieved a maximum accuracy of $99.9 \%$ for binary class and $97.3 \%$ for multi-class. The suggested technique had a greater accuracy of $95.2 \%$ than the CoroNet algorithm, which had an accuracy of $89.88 \%$. The benchmark performance metrics of accuracy, F1-score, precision, recall, and specificity were used to evaluate the performance of proposed system on two different types of classification. Our suggested method can assist medical doctors in making decisions about COVID-19 cases based on CXR images, according to the results of the experimental analysis.

\section{REFERENCES}

[1] B. Sekeroglu and I. Ozsahin, "Detection of COVID-19 from Chest X-Ray Images Using Convolutional Neural Networks," SLAS TECHNOLOGY, vol. 25, pp. 553-565, 2020, doi: 10.1177/2472630320958376.

[2] A. Makris, I. Kontopoulos, and K. Tserpes, "COVID-19 detection from chest X-Ray images using Deep Learning and Convolutional Neural Networks," SETN 2020: 11th Hell. Conf. Arti. Intel., pp. 60-66, 2020, doi: 10.1101/2020.05.22.20110817. 
[3] N. S. Punn and S. Agarwal, " Automated diagnosis of COVID-19 with limited posteroanterior chest X-ray images using fine-tuned deep neural networks," IEEE Transactions on Industrial Electronics, pp. 2689-2702, Oct. 2020, doi: 10.1007/s10489-020-01900-3.

[4] S. S. S. Thejeshwar, C. Chokkareddy, and K. Eswaran, "Precise Prediction of COVID-19 in Chest X-Ray Images Using KE Sieve Algorithm," medRxiv Preprint, pp. 1-5, 2020, doi: 10.1101/2020.08.13.20174144.

[5] M. A. Shereen, S. Khan, A. Kazmi, N. Bashir, and R. Siddique., "COVID-19 infection: Origin, transmission, and characteristics of human corona viruses," Journal of Advanced Research, vol. 24, pp. 91-98, Jul. 2020, doi: 10.1016/j.jare.2020.03.005.

[6] C. B. Cunha and S. M. Opal, "Middle East respiratory syndrome (MERS)," Virulence, vol 5, pp. 650-654, Aug. 2014, doi: 10.4161/Fviru.32077.

[7] M. Nishio, S. Noguchi, H. Matsuo, and T. Murakami, "Automatic classifcation between COVID-19 pneumonia, non-COVID-19 pneumonia, and the healthy on chest X-ray image: combination of data augmentation methods," Scientific reports, pp. 1-6, Oct. 2020, doi: 10.1038/s41598-020-74539-2.

[8] M. F. Hashmi, S. Katiyar, A. G. Keskar, N. D. Bokde, and Z. W. Geem, "Efficient Pneumonia Detection in Chest Xray Images Using Deep Transfer Learning," Diagnostics, vol. 10, pp. 3-23, Jun. 2020, doi: 10.3390/diagnostics 10060417.

[9] V. Taslimitehrani, Guozhu Dong, N. L. Pereira, M. Panahiazar, and J. Pathak, "Developing EHR-driven heart failure risk prediction models using CPXR (Log) with the probabilistic loss function," J. Biomed. Informatics, vol. 60, pp. 260-269, Apr. 2016, doi: 10.1016/j.jbi.2016.01.009.

[10] Z.-P. Liu, L.-Y. Wu, Y. Wang, X.-S. Zhang, and L. Chen, "Prediction of protein-RNA binding sites by a random forest method with combined features," Bioinformatics, vol. 26, no. 13, pp. 1616-1622, 2010, doi: 10.1093/bioinformatics/btq253.

[11] J. H. Chen and J. Z. Lin, "Developing an SVM based risk hedging prediction model for construction material suppliers," Autom. Construct, vol. 19, no. 6, pp. 702-708, Oct. 2010, doi: 10.1016/j.autcon.2010.02.014.

[12] H. Sun, Z. Liu, G. Wang, W. Lian, and J. Ma, "Intelligent Analysis of Medical Big Data Based on Deep Learning," IEEE Access, vol. 7, pp. 142022-142037, 2019, doi: 10.1109/ACCESS.2019.2942937.

[13] D. Ravì et al., "Deep Learning for Health Informatics," in IEEE Journal of Biomedical and Health Informatics, vol. 21, no. 1, pp. 4-21, Jan. 2017, doi: 10.1109/JBHI.2016.2636665

[14] Y. Yu, M. Li, L. Liu, Y. Li, and J. Wang, "Clinical big data and deep learning: Applications, challenges, and future outlooks," Big Data Mining and Analytics, vol. 2, no. 4, pp. 288-305, Dec. 2019, doi: 10.26599/BDMA.2019.9020007.

[15] L. N. Mahdy, K. A. Ezzat, H. H. Elmousalami, H. A. Ella, and A. E. Hassanien, "Automatic X-ray COVID-19 Lung Image Classification System based on Multi-Level Thresholding and Support Vector Machine," medRxiv preprint, Apr. 2020, doi: 10.1101/2020.03.30.20047787.

[16] K. H. Abdulkareem et al., "Realizing an Effective COVID-19 Diagnosis System Based on Machine Learning and IoT in Smart Hospital Environment," IEEE Internet of Things Journal, vol. 8, no. 21, pp. 15919-15928, Nov. 2021, doi: 10.1109/JIOT.2021.3050775.

[17] J. C. Gomes et al.,"IKONOS: an intelligent tool to support diagnosis of COVID-19 by texture analysis of X-ray images," Research on Biomedical Engineering, Aug. 2020, doi: 10.1007\%2Fs42600-020-00091-7.

[18] A. S. Al-Waisy, "COVID-CheXNet: hybrid deep learning framework for identifying COVID-19 virus in chest X-rays images," Soft Computing, Nov. 2020, doi: 10.1007/s00500-020-05424-3.

[19] A. S. Al-Waisy et al., "COVID-DeepNet: Hybrid Multimodal Deep Learning System for Improving COVID-19 Pneumonia Detection in Chest X-ray Images," Computers, Materials and Continua, vol. 67, no. 2, pp. 2409-2429, Feb 2021, doi: $10.32604 / \mathrm{cmc} .2021 .012955$.

[20] L. Wang et al., "COVID-Net: a tailored deep convolutional neural network design for detection of COVID-19 cases from chest X-ray images," Scientific Reports, 10, pp. 1-13, 2020, doi: 10.1038/s41598-020-76550-Z

[21] T. Ozturk, M. Talo, E. A. Yildirim, U. BaranBaloglu, O. Yildirim, and U. R. Acharyafgh, "Automated detection of COVID-19 cases using deep neural networks with X-ray images," Comp. Bio. Med., April 2020, doi: 10.1016/j.compbiomed.2020.103792.

[22] T. Goel, R. Murugan, S. Mirjalili, and D. K. Chakrabartty, "OptCoNet: an optimized convolutional neural network for an automatic diagnosis of COVID-19," Applied Intelligence, pp. 1351-1366, Sept. 2020, doi: 10.1007/s10489-020-01904-Z.

[23] A. I. Khan, J. L. Shah, and M. Mudasir Bhat, "CoroNet: A deep neural network for detection and diagnosis of COVID-19 from chest X-ray images," Computer Methods and Programs in Biomedicine, vol.196, Nov 2020, doi: 10.1016/j.cmpb.2020.105581.

[24] A. Abbas, M. M. Abdelsamea, and M. M. Gaber, "Classification of COVID-19 in chest X-ray images using DeTraC deep convolutional neural network," Applied Intelligence, pp. 854-864, Sep. 2020, doi: 10.1007/s10489-020-01829-7.

[25] M. A. Mohammed et al., "A Comprehensive Investigation of Machine Learning Feature Extraction and Classification Methods for Automated Diagnosis of COVID-19 Based on X-ray Images," Computers, Materials \& Continua, vol. 66, no. 3, Dec. 2021.

[26] Z. A. A. Alyasseri et al., "Review on COVID-19 diagnosis models based on machine learning and deep learning approaches," Expert Systems, Jul. 2021, doi: 10.1111/exsy.12759.

[27] K. He, X. Zhang, S. Ren, and J. Su, "Deep residual learning for image recognition," IEEE Conference on Computer Vision and Pattern Recognition (CVPR), Jun. 2016 doi: 10.1109/CVPR.2016.90.

[28] J. P. Cohen, P. Morrison, and Lan Dao, COVID-19 image data collection," (2020). Accessed: Mar. 25, 2020. [Online]. Available: https://github.com/ieee8023/covid-chestxray-dataset

[29] Chest X-ray images (pneumonia). (2020). Accessed: Apr 1, 2020. [Online]. Available: https://www.kaggle.com/paultimothymooney/chest-xray-pneumonia

[30] G. Yadav, S. Maheshwari, and A. Agarwal, "Contrast limited adaptive histogram equalization-based enhancement for real time video system," 2014 Int. Conf. Adv. Comp., Comm. Inf. (ICACCI), 2014, pp. 2392-2397, doi: 10.1109/ICACCI.2014.6968381.

[31] M. S. Hitam, E. A. Awalludin, W. N. Jawahir Hj Wan Yussof, and Z. Bachok, "Mixture contrast limited adaptive histogram equalization for underwater image enhancement," 2013 International Conference on Computer Applications Technology (ICCAT), 2013, pp. 1-5, doi: 10.1109/ICCAT.2013.6522017.

[32] P. Singh, R. Mukundan, and R. de Ryke, "Feature Enhancement in Medical Ultrasound Videos Using Contrast-Limited Adaptive Histogram Equalization," J Digit Imaging, pp. 273-285, Feb. 2020, doi: 10.1007/s 10278-019-00211-5.

[33] S. B. Patil and B. P. Patil, "Retinal fundus image enhancement using adaptive CLAHE methods, "Journal of Seybold Report, vol. 15, pp. 3476-3484, 2020

[34] N. Salem, H. Malik, and A. Shams, "Medical image enhancement based on histogram algorithms, " Procedia Computer Science ,vol. 163 ,pp. 300-311, 2019, doi: 10.1016/j.procs.2019.12.112

[35] M. Sokolova, N. Japkowicz, and S. Szpakowicz, "Beyond Accuracy, F-Score and ROC: A Family of Discriminant Measures for Performance Evaluation," Australasian joint conf. on artificial intelligence, pp. 1015-1021, 2006, doi: 10.1007/11941439_114.

[36] R. Parikh, A. Mathai, S. Parikh, and G. Chandra Sekhar, "Understanding and using sensitivity, specificity and predictive values," Indian Journal Ophthalmol, pp. 45-50, 2008, doi: 10.4103/0301-4738.37595. 


\section{BIOGRAPHIES OF AUTHORS}
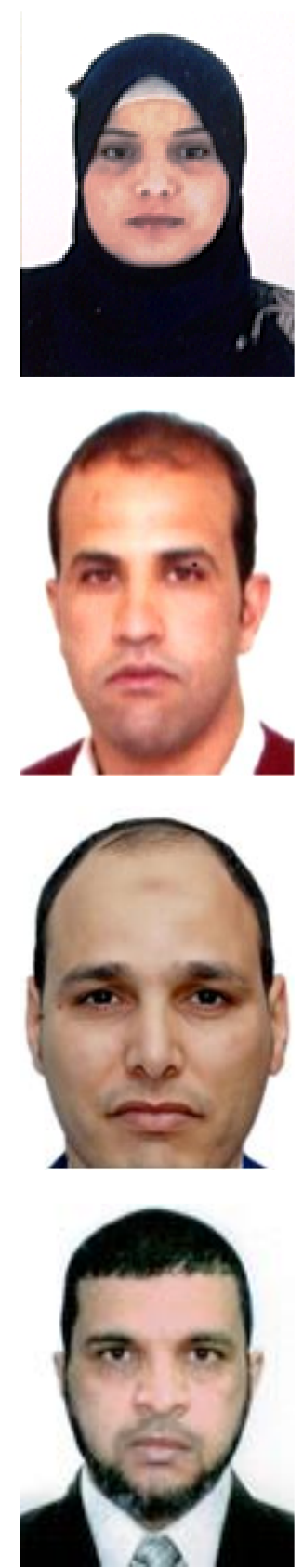

Fatima Zohra Hamlili (iD SC SC P received her bachelor's degree in general electronics from the University TAHRI Mohamed of Bechar (Algeria) in 2013, and her master degree in digital communication system from the University TAHRI Mohamed of Bechar (Algeria) in 2015. Her current research interest includes medical image processing, medical image segmentation, and medical image classification using deep learning. She can be contacted at email: hamlili.fatima@univ-bechar.dz.

Mohammed Beladgham (iD) S SC P Graduated from the University of Tlemcen in Algeria with a diploma in electrical engineering and a master's degree in signals and systems. In 2012, he received his $\mathrm{PhD}$ in Electronics from the University of Tlemcen in Algeria. He is currently a professor at the Department of Electrical Engineering, Faculty of Technology, University TAHRI Mohamed of Bechar, Algeria. His research interests include image segmentation, medical image compression, video processing, and biomedical imaging. He can be contacted at email: beladgham.mohammed@univ-bechar.dz.

Mustapha Khelifi (D) SC S P He received his bachelor's degree in electrical engineering from the University Moulay Tahar Saida in Algeria in 2009, and his master's degree in signal and digital communication from the University TAHRI Mohamed of Bechar in 2011. In 2017, he received his $\mathrm{PhD}$ in telecommunications from Algeria's TAHRI Mohamed of Bechar University. His research interests include compression image, image processing. He can be contacted at email: khelifi.mostepha@univ-bechar.dz.

Ahmed Bouida (iD 8. SC $\mathrm{P}$ He earned his bachelor's degree in electrical engineering and his master's degree in electronics from the University Djillali Liabes of Sidi Bel Abbes in 1994 and 1998, respectively (Algeria). He received his PhD in Electrical Engineering from Algeria's TAHRI Mohamed of Bechar University in 2021. His current research interests include image processing, biometric image compression, first and second-generation wavelets transform, image quality assessment, and image with Deep Learning. He can be contacted at email: bouida.ahmed@univ-bechar.dz. 